\title{
Melatonin suppression by melanopsin-weighted light in patients with bipolar I disorder compared to healthy controls
}

\author{
Philipp Ritter, MD; Falk Wieland, PhD; Debra J. Skene, PhD; Andrea Pfennig, MD; \\ Maria Weiss, MD; Michael Bauer, MD; Emanuel Severus, MD; Henry Güldner, PhD; \\ Cathrin Sauer, DiplBiomath; Bettina Soltmann, MSc; Stefanie Neumann, MD
}

\begin{abstract}
Background: Multiple lines of evidence suggest that the onset and course of bipolar disorder is influenced by environmental light conditions. Increased suppression of melatonin by light (supersensitivity) in patients with bipolar disorder has been postulated as an endophenotype by several studies. However, due to methodological shortcomings, the results of these studies remain inconclusive. This study investigated melatonin suppression in euthymic patients with bipolar I disorder using evening blue light specifically targeting the melanopsin system. Methods: Melatonin suppression was assessed in euthymic patients with bipolar I disorder and healthy controls by exposure to monochromatic blue light $\left(\lambda_{\max }=475 \mathrm{~nm}\right.$; photon density $=1.6 \times 10^{13}$ photons $\left./ \mathrm{cm}^{2} / \mathrm{s}\right)$ for 30 minutes at $2300 \mathrm{~h}$, administered via a ganzfeld dome for highly uniform light exposure. Serum melatonin concentrations were determined from serial blood sampling via radioimmunoassay. All participants received mydriatic eye drops and were genotyped for the PER3 VNTR polymorphism to avoid or adjust for potential confounding. As secondary outcomes, serum melatonin concentrations during dark conditions and after monochromatic red light exposure $\left(\lambda_{\max }=624 \mathrm{~nm}\right.$; photon density $=1.6 \times 10^{13}$ photons $\left./ \mathrm{cm}^{2} / \mathrm{s}\right)$ were also investigated. Changes in subjective alertness were investigated for all 3 lighting conditions. Results: A total of 90 participants (57 controls, 33 bipolar I disorder) completed the study. Melatonin suppression by monochromatic blue light did not differ between groups $\left(F_{1,80}=0.56 ; p=0.46\right)$. Moreover, there were no differences in melatonin suppression by monochromatic red light $\left(F_{1,82}=1.80 ; p=0.18\right)$ or differences in melatonin concentrations during dark conditions $\left(F_{1,74}=1.16 ; p=0.29\right)$. Healthy controls displayed a stronger increase in subjective alertness during exposure to blue light than patients with bipolar I disorder $\left(t_{85}=2.28 ; p=0.027\right)$. Limitations: Large interindividual differences in melatonin kinetics may have masked a true difference. Conclusion: Despite using a large cohort and highly controlled laboratory conditions, we found no differences in melatonin suppression between euthymic patients with bipolar I disorder and healthy controls. These findings do not support the notion that supersensitivity is a valid endophenotype in bipolar I disorder.
\end{abstract}

\section{Introduction}

Bipolar disorder is a severe mental disorder that has a significant negative impact on psychosocial functioning and quality of life; it is associated with high rates of suicide.

There is currently no comprehensive neurobiological model that explains the mechanisms causing, modulating and sustaining bipolar disorder. More recently, however, evidence has accumulated that its onset and course are influenced by alterations in environmental light conditions and aberrant melatonin signalling. ${ }^{1}$ An initial study demonstrating an inverse correlation between the springtime change in light intensity (solar insolation) and age at onset of the disor- der ${ }^{2}$ has been replicated in 2 follow-up studies. ${ }^{3,4}$ Peaks for both manic and depressive episodes appear to follow a seasonal pattern. ${ }^{5}$ Melatonin levels have been found to be reduced in bipolar disorder, ${ }^{6}$ and exposure to sunlight has been found to shorten time to remission in bipolar depression. ${ }^{7}$ Further studies have also demonstrated that restricting light exposure has a therapeutic antimanic effect. ${ }^{8,9}$

The synthesis of melatonin is suppressed by light, particularly in the short-wavelength blue range, ${ }^{10,11}$ and transmitted via neural pathways involving the suprachiasmatic nuclei and the autonomic nervous system innervating the pineal gland. Melatonin plays a central role in modulating sleep timing and synchronizing circadian rhythms, both processes

Correspondence to: P. Ritter, University Hospital Carl Gustav Carus Dresden, Fetscherstrasse 74, 01307 Dresden, Germany; philipp.ritter@ukdd.de

Submitted Jan. 7, 2019; Revised Apr. 12, 2019; Accepted Apr. 12, 2019; Published online Sept. 11, 2019

DOI: $10.1503 / j p n .190005$ 
known to be altered in patients with bipolar disorder. ${ }^{12,13} \mathrm{Re}-$ search in the area of nonvisual light responses has received substantial stimulus from the discovery of the novel photopigment melanopsin and description of intrinsically photosensitive retinal ganglion cells (ipRGCs). ${ }^{14}$ Melanopsincontaining ipRGCs appear to be the main source and gateway for nonvisual information on general levels of environmental light transmitted to the central nervous system. The absorption maximum for melanopsin is estimated to be in the blue spectrum, with a peak at $480 \mathrm{~nm} \cdot{ }^{15,16}$ Aside from its critical role in circadian entrainment and melatonin suppression, ipRGC signalling has been shown to be involved in the pupillary light reflex, arousal and vision itself. ${ }^{17}$

Nonvisual light responses appear to be modulated by a variable number tandem repeat (VNTR) polymorphism on the PER3 gene; the $5 / 5\left(P E R 3^{5 / 5}\right)$ genotype displays a more sensitive response to blue-enriched light with respect to melatonin suppression compared to the $P E R 3^{4 / 4}$ genotype. ${ }^{18}$ Interestingly $P E R 3^{5 / 5}$ has also been shown to be associated with an earlier disease onset in patients with bipolar disorder, further underscoring a possible modulating effect of nonvisual light processing in modifying disease course. ${ }^{19}$

Several studies have investigated non-image-forming functions in patients with bipolar disorder by measuring melatonin suppression by bright white light in the evening or at night, but results have been inconsistent. Some studies ${ }^{6,20-23}$ but not all $^{24,25}$ found increased melatonin suppression in patients with bipolar disorder, leading to the proposition that supersensitivity to light may be an endophenotype ${ }^{21}$ in bipolar I disorder. This view has been enforced by the observation that the moodstabilizing drugs lithium and valproate both reduce melatonin sensitivity to light. ${ }^{26,27}$ However, these studies had a variety of methodical drawbacks: most had small samples of patients with bipolar disorder $(n<20)$, included patients in manic or depressive episodes, and used white light with an undefined spectral composition. Therefore, it remains uncertain whether the increased light sensitivity occurs in the melanopsin (blue) spectrum or in other components of white light (e.g., red). In addition, none of the studies dilated the participants' pupils or used ganzfeld domes, making it impossible to estimate accurately the photon density of the light stimulus and increasing the risk of bias due to medication or illness effects on patients' pupil size. ${ }^{28}$ Moreover, none of the studies have specifically investigated melatonin suppression taking into account the spectral sensitivity of melanopsin and the potentially confounding effect of unequal distribution of the PER3 VNTR genotype.

The primary aim of the present study was to determine whether patients with bipolar I disorder were more sensitive to melatonin suppression by monochromatic blue light $\left(\lambda_{\max }=\right.$ $475 \mathrm{~nm}$ ) specifically stimulating the melanopsin pathway compared with healthy controls.

Our secondary aims were as follows: to determine whether patients with bipolar I disorder were more sensitive to melatonin suppression by narrow-bandwidth red light $\left(\lambda_{\max }=624 \mathrm{~nm}\right)$ compared with healthy controls, to determine whether patients with bipolar I disorder had lower serum melatonin concentrations than healthy controls, and to determine whether patients with bipolar I disorder were more sensitive to the subjective alerting effects of monochromatic blue light $\left(\lambda_{\max }=475 \mathrm{~nm}\right)$ as measured by the Karolinska Sleepiness Scale (KSS). ${ }^{29}$

We also performed exploratory analyses to determine whether the PER3 VNTR genotype was associated with melatonin suppression by monochromatic blue light.

\section{Methods}

\section{Participants}

The study was approved by the institutional review board of the Medical Faculty of the Technische Universität Dresden (IRB00001473 and IORG0001076). We recruited patients with bipolar I disorder from the outpatient departments of 4 hospitals, and by advertisement in local newspapers and the websites of bipolar advocacy groups. We recruited healthy controls via notice boards and advertisements in local newspapers. After providing full informed consent, all participants were assessed using the German version of the SCID I interview ${ }^{30}$ for DSM-IV by an experienced psychiatrist. Mood was assessed using the Young Mania Rating Scale (YMRS) ${ }^{31}$ and the Inventory of Depressive Symptoms (IDS). ${ }^{32}$ Ophthalmological conditions were excluded by slit lamp examination, tonometry, and Snellen and Ishihara charts. We also recorded body mass index, smoking status, current medication, family history of psychiatric disorder and past medical history. Further instruments used to characterize participants' sleep and circadian rhythms were the Pittsburgh Sleep Quality Index (PSQI), ${ }^{33}$ the $\mathrm{KSS}^{29}$ and the MorningnessEveningness Scale (MEQ). ${ }^{34}$ We used the Global Assessment of Functioning (GAF) ${ }^{35}$ to quantify overall functioning.

Healthy control participants were excluded if any axis I psychiatric disorder (excluding adjustment disorder in full remission) or emotionally unstable personality disorder had been diagnosed and if first-degree relatives had been diagnosed or could be assumed to have an affective, substance use, anxiety or psychotic disorder. Patients with bipolar I disorder were excluded if any additional axis I psychiatric disorder or emotionally unstable personality disorder had been diagnosed. Further exclusion criteria for all participants included ophthalmological conditions (except for simple myopia/hyperopia); diagnosed or probable sleeping disorder; other disorders associated with impaired sleep (i.e., polyneuropathy, chronic severe back pain); substance use disorder; inflammatory conditions; neurologic conditions; cancer; medical conditions deemed incompatible with study participation; use of melatonin, pregabalin, gabapentin, benzodiazepines, zopiclone, zolpidem or $\beta$-blockers; recent transmeridian travel (past 2 weeks); or shift or night-time work (past 3 months). All participants were required to be between 18 and 60 years of age and euthymic at the time of inclusion and on every assessment night (YMRS $<5$; IDS $<8$ ). The study was conducted during the winter months (November 2015 to March 2016) at latitude $51^{\circ} \mathrm{N}$.

\section{Study protocol}

Study participants attended the laboratory for assessment on a total of 3 occasions. All participants were required to adhere to a regular schedule of waking no later than $0730 \mathrm{~h}$, exposing 
themselves to sunlight in the morning, and switching lights off no later than $2400 \mathrm{~h}$ on the 4 days leading up to each appointment. These prelaboratory conditions ensured stable circadian entrainment and sufficient sleep before the study. Adherence was ascertained by diary. On the day of assessment, participants were required to consume no food or caffeinated drinks after $1300 \mathrm{~h}$. Participants entered the laboratory at $1800 \mathrm{~h}$ on the evening of the assessment. After clarifying the schedule, evaluating the diary and establishing euthymia via YMRS and IDS, we gave participants a standard vegetarian meal of 600 calories at 1900 h. Thereafter, an 18G intravenous cannula was inserted into a forearm vein and $7.5 \mathrm{~mL}$ of blood was collected into a tube containing EDTA (SMonovette K3, Sarstedt) for genetic analyses on the first night.

At $2000 \mathrm{~h}$, all participants were seated in a reclining position under dim light conditions $(<10$ lux in the direction of gaze, incandescent lamps) and allowed to listen to music via headphones or read. Participants were instructed to keep their eyes open. At 2100 h, lights were turned off and participants' eyes were covered with a standard light-blocking eye mask. At $2200 \mathrm{~h}$, participants received mydriatic eye drops $(0.5 \%$ tropicamide and $1.25 \%$ phenylephrine) ${ }^{36}$ bilaterally under dim light conditions. Participants were instructed to keep their eyes closed between 2100 and 2300 h. During the protocol, they were allowed to consume water or herbal tea, but no food. Complete pupil dilation was assessed by study personnel before light exposure.

Between 2300 and $2330 \mathrm{~h}$, participants were exposed to 1 of 3 light conditions (dark, red, blue). The sequence of light exposure was randomized, and patients were blinded to light condition up to the point of exposure. For a uniform full-field exposure of the retina for all participants, we used a half sphere (ganzfeld dome, Optopolymer) with an inner diameter of $50 \mathrm{~cm}$. The inner surface was coated with a highly reflective barium sulphate coating. For highly uniform light distribution inside the dome, LEDs were mounted inside an aperture ring. Standardized light exposure was ensured by a chin and forehead rest; participants sat in an upright position. Constant retinal illumination was ensured via closed-loop constant current LED drivers and integrated continuous photometric measurement linked to an electronic feedback control (Appendix 1, Figure S1, available at jpn.ca/190005-a1).

The photon densities of the light stimuli were set to be at the midpoint of the sigmoid curve describing melatonin suppression. ${ }^{11}$ Light levels were measured with a dummy face at the position of the eye (vertical, looking into the sphere; for a detailed description, see Appendix 1, Figure S1). The red exposure condition was set at a wavelength of $624 \mathrm{~nm}$ (half bandwidth $18 \mathrm{~nm}$ ) and blue was set at $475 \mathrm{~nm}$ (half bandwidth $25 \mathrm{~nm}$ ) with equal photon densities of $1.6 \times 10^{13}$ photons / $\mathrm{cm}^{2} / \mathrm{s}$ (Table 1). Participants were instructed to keep their eyes open throughout the exposure; this was monitored by the staff. We calculated photoreceptor-specific illuminance values in $\alpha$-opic lux using the Irradiance Toolbox. ${ }^{37}$

At $2330 \mathrm{~h}$, participants reclined in their seat and were subjected to renewed darkness until $0000 \mathrm{~h}$.

Blood samples were taken at 2100, 2200, 2300 (before lights on), 2330 (with lights on) and at $0000 \mathrm{~h}$ (lights off); $5 \mathrm{~mL}$ of

\begin{tabular}{lccc}
\multicolumn{4}{l}{ Table 1: Photometric properties of the 3 illuminance conditions } \\
\hline Photometric property & Dark & Red & Blue \\
\hline Peak wavelength, $\mathrm{nm}$ & - & 624 & 475 \\
Full width at half maximum, $\mathrm{nm}$ & - & 18 & 25 \\
Number of photons $/ \mathrm{cm}^{2} / \mathrm{s}$ & 0 & $1.6 \times 10^{13}$ & $1.6 \times 10^{13}$ \\
Illuminance, lux & 0 & 9.2 & 8.0 \\
Irradiance, W/m & 0 & $5.0 \times 10^{-2}$ & $6.6 \times 10^{-2}$ \\
\hline
\end{tabular}

blood was removed and discarded before each sampling. The intravenous cannula was kept patent with $10 \mathrm{~mL}$ of $0.9 \%$ $\mathrm{NaCl}$ solution.

Patients were asked to rate their subjective sleepiness using the KSS at 2100, 2200, 2300, 2315, 2330 and $0000 \mathrm{~h}$.

\section{Laboratory procedures}

Blood was collected in $9 \mathrm{~mL}$ clotting tubes (S-Monovette Z-Gel, Sarstedt) for melatonin analysis and in tubes containing EDTA (S-Monovette K3, Sarstedt) for genetic analyses. After centrifugation of the clotting tubes at $1000 \mathrm{~g}$ for 15 minutes, the supernatant serum and the EDTA whole blood were stored at $-80^{\circ} \mathrm{C}$ until further analysis.

Serum melatonin concentrations were measured in duplicate using a direct radioimmunoassay with 125I-labelled melatonin according to the manufacturer's protocol (IBL International). Radioactivity was measured in a gamma counter LB2111 (Berthold Technologies).

Genomic DNA was extracted from frozen $\left(-80^{\circ} \mathrm{C}\right)$ EDTA whole blood using a QIAmp DNA blood mini kit (Qiagen) according to the manufacturer's protocol. To quantify and assess the purity of the DNA, the absorbance at $260 \mathrm{~nm}$ and the ratios A 260/280 and A260/230 were measured using a NanoDrop 2000 spectrophotometer (Thermo Fisher).

Polymerase chain reaction (PCR) was performed with the following primers: 5'-TGTCTTTTCATGTGCCCTTACTT-3' and 5'-TGTCTGGCATTGGAGTTTGA-3' ${ }^{\prime 8}$ The PCR reaction was carried out in a $25 \mu \mathrm{L}$ volume containing $150 \mathrm{ng}$ DNA, $0.5 \mu \mathrm{M}$ of each primer, $200 \mu \mathrm{M}$ deoxynucleoside triphosphate, $1 \times$ Q5 reaction buffer and $0.02 \mathrm{U} / \mu \mathrm{L}$ Q5 high-fidelity DNA polymerase (New England Biolabs). After an initial step of 30 seconds at $98^{\circ} \mathrm{C}, 30$ cycles of amplification $(10$ seconds at $98^{\circ} \mathrm{C}, 30$ seconds at $65^{\circ} \mathrm{C}, 25$ seconds at $72^{\circ} \mathrm{C}$ ) and a final elongation step of 5 minutes at $72^{\circ} \mathrm{C}$ were performed. An aliquot of PCR product was analyzed on agarose ( $2 \%$ agarose LE, Biozym) gel electrophoresis ( $5 \mathrm{~V} / \mathrm{cm}$ for 90 minutes) to distinguish between the 5 repeat allele (401 bp), the 4 repeat allele (347 bp) and the heterozygotes (347 and $401 \mathrm{bp}$ ).

\section{Statistical analysis}

We calculated the minimum sample size ${ }^{39}$ based on previous publications ${ }^{6}$ with mean melatonin suppression (bipolar I disorder $62 \%$, healthy controls $40 \%$, standard deviation $15 \%$; $n=$ 14 for $1-\beta=0.8, \alpha=5 \%$ ).

We compared demographic and clinical characteristics using 2 -sided unpaired $t$ tests and $\chi^{2}$ tests as appropriate. In 
Table 2: Demographic and clinical characteristics of healthy controls and patients with bipolar I disorder

\begin{tabular}{|c|c|c|c|}
\hline Characteristic & $\begin{array}{l}\text { Healthy } \\
\text { controls } \\
(n=57)\end{array}$ & $\begin{array}{l}\text { Bipolar I } \\
\text { disorder } \\
(n=33)\end{array}$ & $p$ value $^{\star}$ \\
\hline Age, yr, mean $\pm S D$ & $39.6 \pm 9.6$ & $43.3 \pm 10.9$ & 0.09 \\
\hline Female, \% & 63.2 & 57.6 & 0.66 \\
\hline PSQI score, mean \pm SD & $3.3 \pm 1.9$ & $6.0 \pm 4.0$ & $<0.001$ \\
\hline MEQ score, mean \pm SD & $56.8 \pm 9.5$ & $51.0 \pm 11.8$ & 0.017 \\
\hline GAF score, mean \pm SD & $99.5 \pm 1.7$ & $83.0 \pm 10.6$ & $<0.001$ \\
\hline $\begin{array}{l}\text { Qualified for higher } \\
\text { education, \% }\end{array}$ & 65.5 & 72.0 & $0.36 \dagger$ \\
\hline Positive family history, \%‡ & 0 & 24.2 & \\
\hline \multicolumn{4}{|l|}{ Current medication, \% } \\
\hline Lithium & - & 54.6 & - \\
\hline Valproate & - & 9.1 & - \\
\hline Lamotrigine & - & 18.2 & - \\
\hline Olanzapine & - & 9.1 & - \\
\hline Quetiapine & - & 18.2 & - \\
\hline $\begin{array}{l}\text { Other atypical } \\
\text { antipsychotic }\end{array}$ & - & 12.1 & - \\
\hline Antidepressant & - & 15.2 & - \\
\hline
\end{tabular}

$\mathrm{GAF}=$ Global Assessment of Functioning; $\mathrm{MEQ}=$ Morningness-Eveningness

Questionnaire; PSQI = Pittsburgh Sleep Quality Index; SD = standard deviation

*2-tailed $t$ test, unless stated otherwise.

$\dagger \chi^{2}$ test.

$\ddagger$ Parental history of bipolar disorder or schizophrenia.

cases of significant differences between the bipolar I disorder and healthy control groups, we included potential relevant confounders in the analysis as covariates.

We made groupwise comparisons following visual inspection of Q/Q plots and assessment of normal distribution using the Shapiro-Wilk test. We used $t$ tests, Mann-Whitney tests, repeated-measures analyses of covariance (ANCOVA) and univariate ANCOVA as appropriate to compare differences in outcomes between the bipolar I disorder and healthy control groups.

The primary outcomes were relative and absolute suppression of serum melatonin concentration 30 minutes after the beginning of exposure to blue light. Relative suppression was calculated as ([melatonin at $2300 \mathrm{~h}$ - melatonin at $2330 \mathrm{~h}]$ / melatonin at $2300 \mathrm{~h}) \times 100$. We also calculated melatonin suppression by exposure to red light. We evaluated group differences in relative and absolute suppression using univariate ANCOVA with age and chronotype (MEQ score) as covariates.

We evaluated differences in total melatonin concentration between patients using repeated-measures ANOVA adjusted for age and chronotype.

We evaluated the differential effects of group on changes in KSS during exposure to blue light using repeated-measures ANCOVA with age and MEQ as covariates. We performed post hoc $t$ tests for selected time points.

We calculated differences in distribution of PER3 VNTR genotype between the healthy control and bipolar I disorder groups using $\chi^{2}$ tests.

We evaluated the relationship between melatonin suppression and demographic and clinical characteristics using

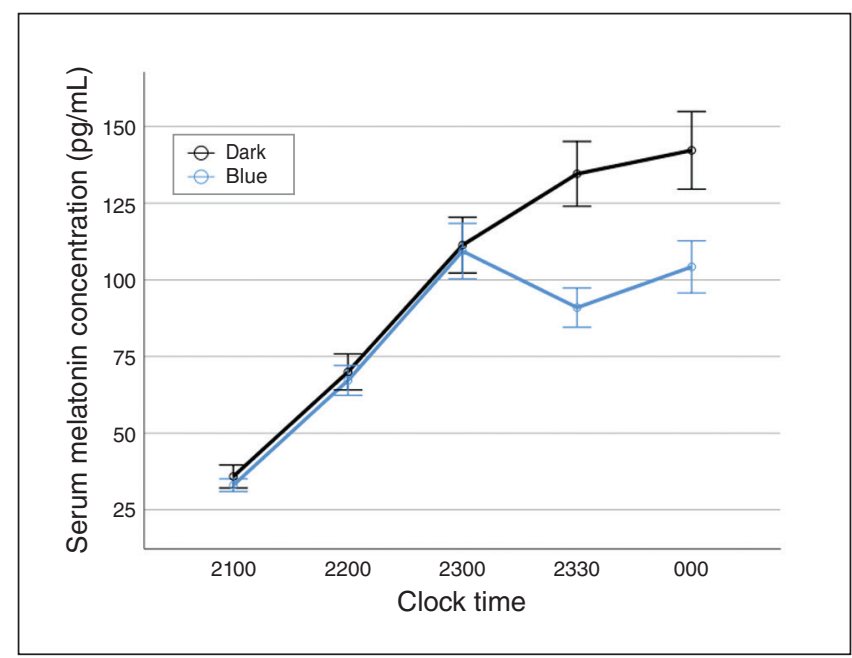

Fig. 1: Serum melatonin concentration $(\mathrm{pg} / \mathrm{mL})$ for all participants ( $n=90)$ comparing conditions of no light exposure (dark; black line) with exposure to narrow-bandwidth blue light (blue line) between 2300 and $2330 \mathrm{~h}$. Error bars indicate standard error of the mean.

Pearson or Spearman correlation coefficients as appropriate. We assessed the effect of mood-stabilizing medication using $t$ tests or repeated-measures ANOVA, comparing patients receiving mood-stabilizers (lithium or valproate; $n=19$ ) to those receiving other medications $(n=11)$.

\section{Results}

A total of 116 participants were screened for inclusion in the study. Of these, 94 completed at least 1 assessment and 90 completed all 3 assessments. Only participants completing all 3 study nights were included in the analysis. Of the healthy controls, 6 declined to participate following screening, 2 did not respond to further communication, 2 discontinued after 1 study night because of poor tolerability, 2 had a positive family history, 1 was consuming cannabis and 1 was found to be red-green colour-blind. Among bipolar I disorder participants, 5 declined following screening, 2 discontinued after 1 study night because of poor tolerability, 3 did not respond to further communication, 2 did not fulfill the diagnostic criteria for bipolar I disorder. The participants in the 2 study groups were well matched for age and sex (Table 2).

\section{Melatonin suppression}

Narrow-bandwidth blue light led to a measurable relative suppression in evening melatonin $(11 \%$; standard deviation 29; Fig. 1). We found no significant differences between patients with bipolar I disorder and healthy controls for either relative $\left(F_{1,80}=0.56 ; p=0.46\right)$ or absolute $\left(F_{1,80}=0.0 ; p=\right.$ $0.99)$ suppression of melatonin by monochromatic blue light (Fig. 2; Appendix 1, Figure S4).

We also found no difference between the 2 groups with respect to relative $\left(F_{1,82}=1.80 ; p=0.18\right)$ or absolute $\left(F_{1,82}=\right.$ 


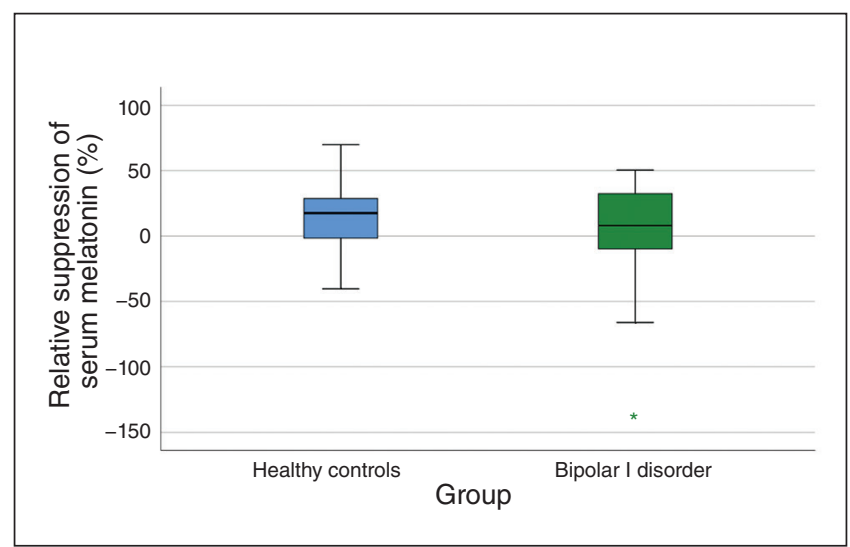

Fig. 2: Box plots of suppression of serum melatonin by narrowbandwidth blue light. Positive values denote suppression $(2330 \mathrm{~h})$ of melatonin relative to baseline $(2300 \mathrm{~h})$.

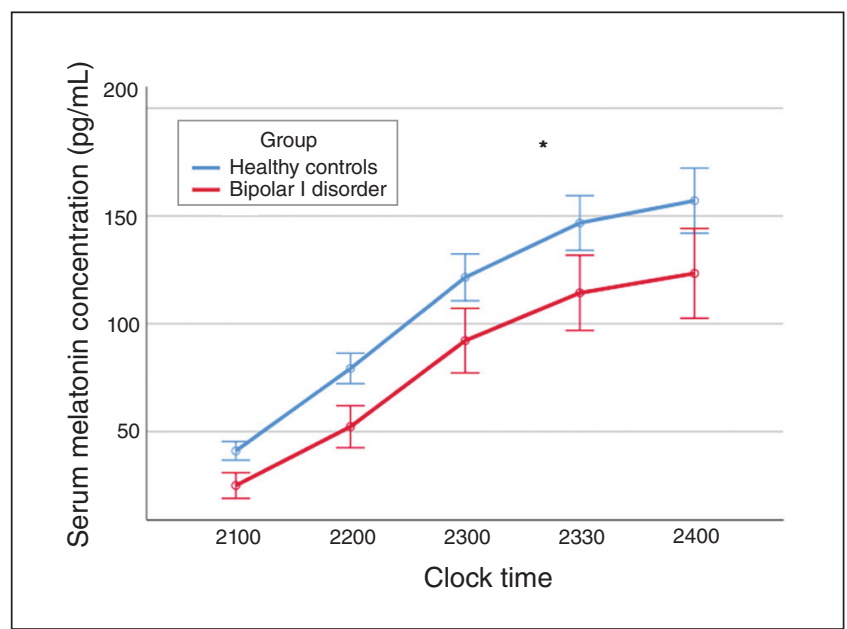

Fig. 4: Serum melatonin concentration $(\mathrm{pg} / \mathrm{mL})$ with no light exposure comparing patients with bipolar I disorder and healthy controls. Error bars indicate standard error of the mean.

0.64; $p=0.43$ ) melatonin suppression by monochromatic red light (Fig. 3; Appendix 1, Figure S4).

Participants with bipolar disorder had reduced serum melatonin levels compared with healthy controls during dark conditions, but this finding was not statistically significant after adjusting for age and chronotype $\left(F_{1,74}=1.16 ; p=\right.$ 0.29; Fig. 4).

Participants with bipolar I disorder were more likely to be evening types (as assessed by the MEQ) compared with healthy controls $\left(t_{82}=2.44 ; p=0.017\right.$; Table 2$)$, but there were no significant correlations between MEQ and outcome parameters (Appendix 1, Table S6). Participants with bipolar I disorder also described a worse quality of subjective sleep (as assessed by PSQI) compared to healthy controls $\left(t_{88}=4.39 ; p<0.001\right)$

The use of lithium or valproate did not significantly affect melatonin concentrations $\left(F_{1,31}=0.44 ; p=0.52\right)$ or relative $\left(t_{28}=\right.$ $0.81 ; p=0.42)$ or absolute $\left(t_{28}=0.04 ; p=0.97\right)$ melatonin suppression by blue light (Appendix 1, Table S2).

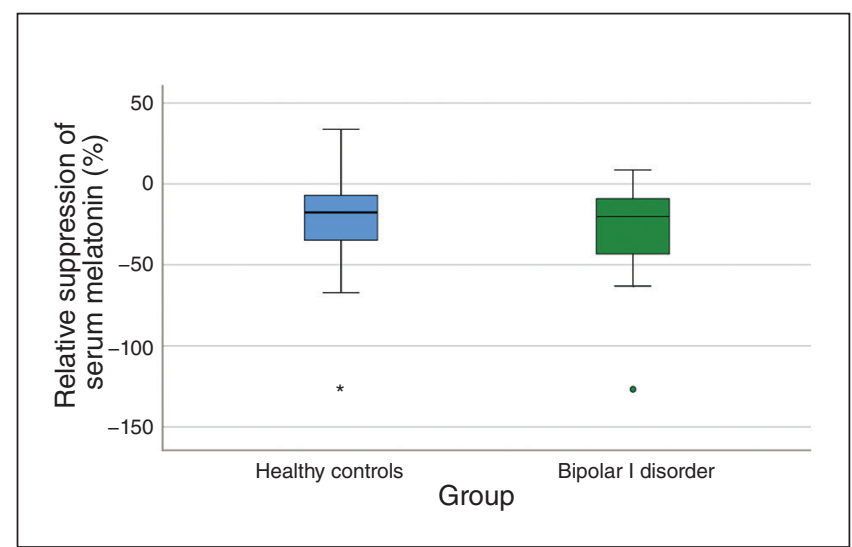

Fig. 3: Box plots of suppression of serum melatonin by narrowbandwidth red light. Positive values denote suppression (2330 h) of melatonin relative to baseline $(2300 \mathrm{~h})$.

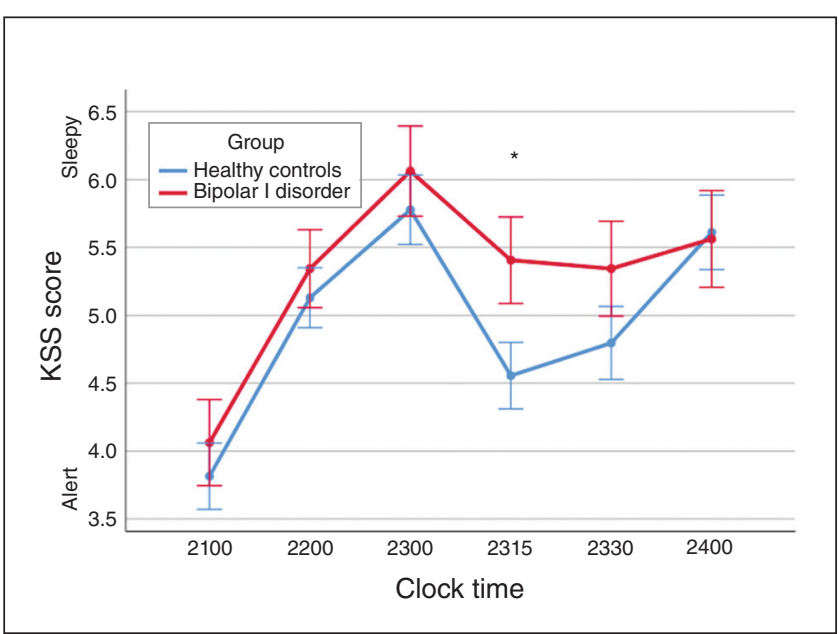

Fig. 5: Karolinska Sleepiness Scale (KSS) score during narrowbandwidth blue light exposure between 2300 and $2330 \mathrm{~h}$ comparing patients with bipolar I disorder and healthy controls. The bipolar I disorder group showed a significantly reduced alerting response to the blue light stimulus compared with healthy controls $\left({ }^{*} p<0.05\right)$. Error bars indicate standard error of the mean.

Using Pearson correlations and $t$ tests, we found no significant associations between relative melatonin suppression and age at onset of disorder $\left(r_{31}=-0.2 ; p=0.2\right)$, number of suicide attempts $\left(r_{31}=-0.06 ; p=0.76\right)$, positive family history for bipolar I disorder $\left(t_{28}=0.72 ; p=0.48\right)$, GAF $\left(r_{31}=-0.1 ; p=\right.$ $0.74)$ or PSQI $\left(r_{31}=0.1 ; p=0.59\right)$.

\section{Subjective alerting response}

Patients with bipolar I disorder had nonsignificantly elevated KSS values $\left(F_{1,79}=2.04 ; p=0.16\right)$ during the dark condition, indicating higher degrees of sleepiness than healthy controls. They also displayed a significantly reduced subjective alerting response (post hoc $t_{85}=2.28 ; p=0.027$ ) during exposure to blue light (Fig. 5). 


\section{PER3 VNTR polymorphism}

The observed genotype frequencies of our sample were $43.7 \%$ $\left(P E R 3^{4 / 4}\right), 40.6 \%\left(P E R 3^{4 / 5}\right)$ and $15.6 \%\left(P E R 3^{5 / 5}\right)$ for patients with bipolar I disorder and $41.5 \%\left(P E R 3^{4 / 4}\right), 49.1 \%\left(P E R 3^{4 / 5}\right)$ and 9.4\% $\left(\right.$ PER $3^{5 / 5)}$ for healthy controls. The PER3 VNTR polymorphism was equally distributed between participants with bipolar I disorder and healthy controls $\left(\chi_{2}^{2}=0.95 ; p=0.62\right)$.

The PER3 VNTR genotype did not correlate with relative melatonin suppression $\left(F_{1,80}=0.61 ; p=0.55\right)$ or melatonin concentration $\left(F_{1,75}=0.07 ; p=0.94\right)$.

\section{Discussion}

\section{Melatonin and melatonin suppression}

Contrary to previous publications $\mathrm{s}^{6,20-23}$ and a widespread assumption in the literature, ${ }^{1,40}$ we did not observe a statistically significant increase in light-induced melatonin suppression or reduced levels of serum melatonin in patients with bipolar I disorder compared with matched healthy controls. Our study tested the largest sample of participants published to date and carefully controlled for factors known to confound melatonin assessment. In addition, it was the only study that investigated bipolar patients with dilated pupils, that restricted investigations to the winter months, and that used ganzfeld domes and monochromatic blue light to optimally stimulate the melanopsin-ipRGC pathway. As well, to the best of our knowledge this was the only study to take into account the effects of a genetic variant that modulates melatonin suppression. Several explanations might account for the discrepancy between our study and the previous reports.

First, the previously reported hypersensitivity of patients with bipolar disorder to evening light exposure may be attributable to components of the visible spectrum that were not explored in our study, including green light components at $530 \mathrm{~nm}$, which was stimulated only with $20 \alpha$-opic lux under blue light conditions (Appendix 1, Table S8). Because other studies did not specify the spectral composition of the light sources in detail, we cannot rule out the possibility that the studies describing a hypersensitivity primarily exposed patients with bipolar I disorder to this wavelength range. Based on the now well-established physiology of melatonin suppression by light in the blue wavelength range, ${ }^{10,11,18}$ this explanation seems unlikely, but not impossible.

Second, mood-stabilizing medication may have disguised a true difference in light-induced melatonin suppression. Both lithium and valproate have been found to reduce lightinduced melatonin suppression in healthy volunteers. ${ }^{26,41} \mathrm{Be}-$ cause a substantial number of our bipolar I disorder participants were receiving either lithium or valproate, true differences may have been masked. Moreover, the study by Nurnberger and colleagues ${ }^{6}$ found an increase in lightinduced melatonin suppression in patients depending on the period of medication discontinuation. However, the 2 studies showing no abnormal response to light in bipolar I disorder were conducted in participants free of psychotropic medication. ${ }^{24,25}$ In addition, in our study sample, neither lithium nor valproate was found to significantly reduce melatonin suppression. Therefore, mood-stabilizing medication may account for some but not all of the discrepancies.

Third, previous studies have not used mydriatic eye drops to assess melatonin suppression. Pupil dilation allows the photon density of the light stimulus - the main predictor of melatonin suppression - to be estimated accurately. Moreover, group and age differences in average pupil size may have contributed to observed differences in the past. In a separate study, we found patients with bipolar disorder to have marginally larger pupils than healthy controls (unpublished data) during light exposure. This may have been due to the disease itself or an adverse effect of medication. In particular, medications with anticholinergic properties are known to have mydriatic effects. The previously described supersensitivity of bipolar patients may thus be related to differences in pupil diameter.

Fourth, studies suggesting light supersensitivity in bipolar I disorder have generally also used longer periods of light exposure (60 to 120 minutes) and started light exposure after $0000 \mathrm{~h}$. Because light-induced melatonin suppression is affected by the time of light administration, ${ }^{42}$ this may be a reason for the differences in results; however, no obvious neuroendocrine mechanism would plausibly explain these differences. Duration of light exposure ${ }^{43}$ also differed between studies, and this may have had a relevant effect. Because melatonin is metabolized by hepatic P450 enzymes ${ }^{44}$ and has a halflife of approximately 40 minutes, the enzyme-inducing or -inhibiting effects of pharmacotherapy may have masked a true effect that would have become apparent after longer exposure.

Finally, elevated melatonin suppression may describe only a subgroup of patients with bipolar disorder; study results may vary depending on the proportion of this subgroup in the sample. However, correlations with clinical parameters did not help us identify such a subgroup in our study sample.

\section{Measure of subjective alertness}

Contrary to our hypothesis, participants with bipolar disorder did not report an increase in subjective alertness during exposure to blue light. In contrast, during the midpoint of blue light administration, patients with bipolar I disorder reported a significant reduction in subjective alertness as measured by the KSS compared with healthy controls. Recent findings related to the beneficial therapeutic effects of blue light deprivation during episodes of mania ${ }^{8,9}$ could suggest a hyperactive alerting response to blue light in patients with bipolar disorder. Because the KSS has not been used previously in patients with bipolar disorder, and subjective ratings of alertness have a high degree of random variability, these results should be treated with caution until they are replicated in an independent sample.

\section{PER3 VNTR}

Participants (healthy controls and bipolar I disorder) with a PER3 VNTR 5/5 genotype did not exhibit an increase in lightinduced melatonin suppression (Appendix 1, Figure S3, Table S7) in contrast to an earlier study of healthy participants. ${ }^{18}$ 


\section{Limitations}

Although this was the largest study of melatonin light sensitivity $(n=90)$ in people with bipolar I disorder to date, we observed large interindividual differences in melatonin kinetics. Participants were required to adhere to a regular light/dark and sleep/ wake schedule before the study nights, but adherence was not verified by objective measures such as actigraphy. Not all participants exhibited a clear evening surge in melatonin, and this may have been attributable in part to a phase-delayed lifestyle. Differences between groups in light exposure in the days preceding the study could also have had a direct influence on melatonin suppression, which has been shown to be sensitive to light history. ${ }^{44}$ Future studies could reduce variance by expanding the sampling protocol over several days in controlled lighting conditions. Nonetheless, in a population of largely employed participants, the feasibility for this would be challenging.

\section{Conclusion}

There is widespread evidence that the onset and course of bipolar disorder is determined to a certain degree by environmental light conditions. Our findings in euthymic bipolar patients suggest that the mediating mechanism is unlikely to be related to an increased suppression of melatonin by melanopsin-weighted light. In view of the growing knowledge regarding nonvisual neuroanatomical pathways in the central nervous system ${ }^{17,45}$ that project to areas other than the suprachiasmatic nuclei and have been shown to alter brain states on a different time scale, ${ }^{46}$ other avenues of research using electrophysiological or functional MRI measures to assess the effect of light on alertness or emotion processing should be pursued in this patient group.

Acknowledgements: The authors thank Dorothy Ritter for her linguistic contributions to this manuscript and Gerd Wunderlich, Rupert Overall and Klaus Fabel for providing the required laboratory facilities and assistance.

Affiliations: From the Department of Psychiatry and Psychotherapy, University Hospital Carl Gustav Carus, Technische Universität Dresden, Dresden, Germany (Ritter, Wieland, Pfennig, Weiss, Bauer, Severus, Sauer, Soltmann, Neumann); the Chair of Power Electronics, Institute of Electrical Power Engineering, TU Dresden (Wieland and Güldner); and the Department of Chronobiology, Faculty of Health and Medical Sciences, University of Surrey, Guilford, UK (Skene).

Funding: The project was funded by the German Ministry of Education and Science (Bundesministerium für Bildung und Forschung; BMBF), Grant 13N13399.

Competing interests: D. Skene is a co-director of Stockerland Ltd., UK. M. Bauer reports grants from Deutsche Forschungsgemeinscheaft (DFG), and Bundesministerium für Bildung und Forschung (BMBF); personal fees from Allergan, Aristo, Janssen, Lundbeck, Neuraxpharm, Sandoz, Servier and Sumitomo Dainippon; and nonfinancial support from Lilly, Neuraxpharm and Servier, all outside the submitted work. B. Soltmann is supported by a grant from the German Ministry of Research and Education (grant no. 13N13399). No other competing interests declared.

Contributors: P. Ritter, F. Wieland, D. Skene, A. Pfennig, H. Güldner, C. Sauer, B. Soltmann, and S. Neumann designed the study. P. Ritter, F. Wieland, M. Weiss, H. Güldner, C. Sauer, B. Soltmann, and S. Neumann acquired the data, which P. Ritter, F. Wieland, D. Skene,
M. Bauer, E. Severus, H. Gülder, B. Soltmann and S. Neumann analyzed. P. Ritter, F. Wieland, C. Sauer, B. Soltmann, and S. Neumann wrote the article, which P. Ritter, D. Skene, A. Pfennig, M. Weiss, M. Bauer, E. Severus, H. Güldner, and B. Soltmann reviewed. All authors approved the final version to be published and can certify that no other individuals not listed as authors have made substantial contributions to the paper.

\section{References}

1. Etain B, Dumaine A, Bellivier F, et al. Genetic and functional abnormalities of the melatonin biosynthesis pathway in patients with bipolar disorder. Hum Mol Genet 2012;21:4030-7.

2. Bauer M, Glenn T, Alda M, et al. Impact of sunlight on the age of onset of bipolar disorder. Bipolar Disord 2012;14:654-63.

3. Bauer M, Glenn T, Alda M, et al. Relationship between sunlight and the age of onset of bipolar disorder: an international multisite study. J Affect Disord 2014;167:104-11.

4. Bauer M, Glenn T, Alda M, et al. Influence of light exposure during early life on the age of onset of bipolar disorder. J Psychiatr Res 2015;64:1-8.

5. Geoffroy PA, Bellivier F, Scott J, et al. Seasonality and bipolar disorder: a systematic review, from admission rates to seasonality of symptoms. J Affect Disord 2014;168:210-33.

6. Nurnberger JI Jr, Adkins S, Lahiri DK, et al. Melatonin suppression by light in euthymic bipolar and unipolar patients. Arch Gen Psychiatry 2000;57:572-9.

7. Benedetti F, Colombo C, Barbini B, et al. Morning sunlight reduces length of hospitalization in bipolar depression. J Affect Disord 2001;62:221-3.

8. Barbini B, Benedetti F, Colombo C, et al. Dark therapy for mania: a pilot study. Bipolar Disord 2005;7:98-101.

9. Henriksen TE, Skrede S, Fasmer OB, et al. Blue-blocking glasses as additive treatment for mania: a randomized placebo-controlled trial. Bipolar Disord 2016;18:221-32.

10. Brainard GC, Hanifin JP, Greeson JM, et al. Action spectrum for melatonin regulation in humans: evidence for a novel circadian photoreceptor. J Neurosci 2001;21: 6405-12.

11. Thapan K, Arendt J, Skene D. An action spectrum for melatonin suppression: evidence for a novel non-rod, non-cone photoreceptor system in humans. J Physiol 2001;15:261-7.

12. Cajochen C, Chellappa SL, Schmidt C. Circadian and light effects on human sleepiness-alertness. In: Garbarino S, Nobili L, Costa G, editors. Sleepiness and human impact assessment. Milan: Springer Milan; 2014:9-22.

13. Münch M, Kobialka S, Steiner R, et al. Wavelength-dependent effects of evening light exposure on sleep architecture and sleep EEG power density in men. Am J Physiol Regul Integr Comp Physiol 2006;290:R1421-8.

14. Hattar S, Liao H-W, Takao M, et al. Melanopsin containing retinal ganglion cells: architecture, projections, and intrinsic photosensitivity. Science 2002;295:1065-70.

15. Brown TM, Allen AE, al-Enezi J, et al. The melanopic sensitivity function accounts for melanopsin-driven responses in mice under diverse lighting conditions. PLoS One 2013;8:e53583.

16. Lucas RJ, Douglas RH, Foster RG. Characterization of an ocular photopigment capable of driving pupillary constriction in mice. Nat Neurosci Nature Publishing Group 2001;4:621.

17. Hughes S, Jagannath A, Rodgers J, et al. Signalling by melanopsin (OPN4) expressing photosensitive retinal ganglion cells. Eye (Lond) 2016;30:247-54.

18. Chellappa SL, Viola AU, Schmidt C, et al. Human melatonin and alerting response to blue-enriched light depend on a polymorphism in the clock gene PER3. J Clin Endocrinol Metab 2012;97:E433-7.

19. Benedetti F, Dallaspezia S, Colombo C, et al. A length polymorphism in the circadian clock gene Per3 influences age at onset of bipolar disorder. Neurosci Lett 2008;445:184-7. 
20. Nathan PJ, Burrows GD, Norman TR. Melatonin sensitivity to dim white light in affective disorders. Neuropsychopharmacology 1999;21:408-13.

21. Hallam KT, Begg DP, Olver JS, et al. Abnormal dose-response melatonin suppression by light in bipolar type I patients compared with healthy adult subjects. Acta Neuropsychiatr 2009;21:246-55.

22. Lewy A, Wehr T, Goodwin F, et al. Manic-depressive patients may be supersensitive to light. Lancet 1981;317:383-4.

23. Lewy AJ, Nurnberger JI, Wehr TA, et al. Supersensitivity to light: possible trait marker for manic-depressive illness. Am J Psychiatry1985;142:725-7.

When you purposely

prescribe the original brand RELPAX, help make sure your patient gets it.

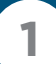

Write the Pfizer original brand name, RELPAX, and include "no sub", when appropriate, on your patient's prescription.

\section{2}

Remind your patient to ask for the original brand RELPAX at the pharmacy.

\section{3}

Encourage your patient

to visit the Pfizer Originals

Program website at

PfizerOriginals.ca

for tips on how to ask

for their Pfizer original

brand medication.

24. Whalley LJ, Perini T, Shering A, et al. Melatonin response to bright light in recovered, drug-free, bipolar patients. Psychiatry Res 1991;38:13-9.

25. Lam RW, Berkowitz AL, Berga SL, et al. Melatonin suppression in bipolar and unipolar mood disorders. Psychiatry Res 1990;33:129-34.

26. Hallam KT, Olver JS, Horgan JE, et al. Low doses of lithium carbonate reduce melatonin light sensitivity in healthy volunteers. Int J Neuropsychopharmacol 2005;8:255-9.

27. Hallam KT, Olver JS, Norman TR. Effect of sodium valproate on nocturnal melatonin sensitivity to light in healthy volunteers. Neuropsychopharmacology 2005;30:1400-4.

28. Siegle GJ, Granholm E, Ingram RE, et al. Pupillary and reaction time measures of sustained processing of negative information in depression. Biol Psychiatry 2001;49:624-36.

29. Åkerstedt T, Gillberg M. Subjective and objective sleepiness in the active individual. Int J Neurosci 1990;52:29-37.

30. Wittchen H-U, Fydrich T, Zaudig M, et al. SKID: Strukturiertes Klinisches Interview für DSM-IV Achse I und II. Achse II : Persönlichkeitsstörungen. SKID-II. Göttingen: Hogrefe; 1997.

31. Young RC, Biggs JT, Ziegler VE, et al. A rating scale for mania: reliability, validity and sensitivity. Br J Psychiatry 1978;133:429-35.

32. Rush AJ, Gullion CM, Basco MR, et al. The inventory of depressive symptomatology (IDS): psychometric properties. Psychol Med 1996;26:477-86.

33. Buysse DJ, Reynolds CF III, Monk TH, et al. The Pittsburgh Sleep Quality Index: a new instrument for psychiatric practice and research. Psychiatry Res 1989;28:193-213.

34. Horne JA, Ostberg O. A self-assessment questionnaire to determine morningness-eveningness in human circadian rhythms. Int J Chronobiol 1976;4:97-110.

35. Spitzer RL, Gibbon JB, Williams W, et al. Global Assessment of Functioning (GAF) scale. Outcomes Assess Clin Pract 1996;76-8.

36. Krumholz DM, Portello JK, Rosenfield M, et al. A combination solution for routine pupillary dilation. Optometry 2006;77:350-3.

37. Lucas RJ, Peirson SN, Berson D, et al. Irradiance Toolbox user guide. Vienna: CIE; 2013. Available: http:/ / files.cie.co.at/784_TN003_Toolbox.xls (accessed 2019 Sep. 3).

38. Artioli P, Lorenzi C, Pirovano A, et al. How do genes exert their role? Period 3 gene variants and possible influences on mood disorder phenotypes. Eur Neuropsychopharmacol 2007;17:587-94.

39. Chow S-C, Wang H, Shao J. Sample size calculations in clinical research. Boca Raton (FL): CRC Press; 2007.

40. Gaspar L, van de Werken M, Johansson AS, et al. Human cellular differences in cAMP: CREB signaling correlate with light-dependent melatonin suppression and bipolar disorder. Eur J Neurosci 2014;40:2206-15

41. Hallam KT, Olver JS, Norman TR. Effect of sodium valproate on nocturnal melatonin sensitivity to light in healthy volunteers. Neuropsychopharmacology 2005;30:1400-4.

42. McIntyre IM, Norman TR, Burrows GD, et al. Human melatonin response to light at different times of the night. Psychoneuroendocrinology 1989;14:187-93.

43. Lockley SW, Brainard GC, Czeisler CA. High sensitivity of the human circadian melatonin rhythm to resetting by short wavelength light. J Clin Endocrinol Metab 2003;88:4205-5.

44. Carpentieri A, Díaz De Barboza G, Areco V, et al. New perspectives in melatonin uses. Pharmacol Res 2012;65:437-44.

45. Lazzerini Ospri L, Prusky G, Hattar S. Mood, The circadian system, and melanopsin retinal ganglion cells. Annu Rev Neurosci 2017;40:539-56.

46. Gaggioni G, Maquet P, Schmidt C, et al. Neuroimaging, cognition, light and circadian rhythms. Front Syst Neurosci 2014;8:1-12.

\section{Pfizer originals ${ }_{\text {program }}{ }$}

